ESJ Humanities

\title{
Enjeux Liés à la Traversée de la Frontière Bénino-Togolaise dans le Secteur Materi-Mandouri
}

\author{
Bakary Sanou
}

Laboratoire de Géographie Rurale et d'Expertise Agricole/UAC, Bénin GIZ : Appui au Programme Frontière de l'Union Africaine

\section{Abdou Madjidou Maman Tondro}

Sylvestre Bio Dakou

Laboratoire de Géographie Rurale et d'Expertise Agricole/UAC, Bénin Youssoufou Adam

Agence Béninoise de Gestion Intégrée des Espaces Frontaliers, Bénin Moussa Gibigaye

Laboratoire de Géographie Rurale et d'Expertise Agricole/UAC, Bénin

Doi:10.19044/esj.2021.v17n42p86

Submitted: 17 August 2021

Accepted: 25 December 2021

Published: 31 December 2021
Copyright 2021 Author(s)

Under Creative Commons BY-NC-ND

4.0 OPEN ACCESS

Cite As:

Sanou B., Maman Tondro A.M., Dakou S.B., Adam Y. \& Gibigaye M. (2021). Enjeux Liés à la Traversée de la Frontière Bénino-Togolaise dans le Secteur Materi-Mandouri. European Scientific Journal, ESJ, 17 (42), 86. https://doi.org/10.19044/esj.2021.v17n42p86

\section{Résumé}

Les zones frontalières sont des lieux spécifiques où s'effectuent des échanges des biens et des personnes. La présente recherche a pour objectif d'étudier les enjeux liés à la traversée de la frontière bénino-togolaise dans le secteur Materi-Mandouri. Pour atteindre cet objectif, des investigations ont été faites à partir de la collecte des données et informations aux moyens de la recherche documentaire, des observations directes sur le terrain, des entretiens et enquêtes auprès de 93 personnes du milieu de recherche choisies suivant la méthode de choix raisonné. Le traitement des données et l'analyse des résultats se sont focalisés sur l'analyse sociale et l'évaluation des gains auprès des populations cibles. Il ressort des résultats que le commerce $(86,66 \%)$, le transport $(6,67 \%)$ et la visite des parents $(6,66 \%)$ sont les principaux facteurs qui expliquent la présence d'un important flux de personnes dans le secteur de recherche. Aussi, les échanges transfrontaliers entre le Bénin et le Togo contribuent au développement de l'économie locale à travers le chargement, le transport de marchandise et les taxes perçues par les autorités locales. 
Cependant, les échanges transfrontaliers sont marqués par des contraintes d'ordre social, sécuritaire et infrastructurel. Il faut alors se poser la question de savoir si les actions actuelles permettent de tirer profit du potentiel économique de cette zone frontalière et de faciliter la traversée des populations.

Mots-clés : Bénin, Togo, frontière, enjeux

\title{
Issues Related to the Crossing of the Benin-Togolese Border in the Materi-Mandouri Sector
}

\author{
Bakary Sanou
}

Laboratoire de Géographie Rurale et d'Expertise Agricole/UAC, Bénin GIZ : Appui au Programme Frontière de l'Union Africaine

\section{Abdou Madjidou Maman Tondro Sylvestre Bio Dakou}

Laboratoire de Géographie Rurale et d'Expertise Agricole/UAC, Bénin Youssoufou Adam

Agence Béninoise de Gestion Intégrée des Espaces Frontaliers, Bénin Moussa Gibigaye

Laboratoire de Géographie Rurale et d'Expertise Agricole/UAC, Bénin

\section{Abstract}

Border areas are specific places where people's goods are traded. The objective of this research is to study the issues related to the crossing of the Benin-Togolese border in the Materi-Mandouri sector. To achieve this objective, investigations were carried out from the collection of data and information by means of documentary research, direct observations in the field, interviews and surveys with 93 people from the research community chosen according to the method of reasoned choice. Data processing and outcome analysis focused on social analysis and evaluation of gains among target populations. The results show that trade $(86.66 \%)$, transport $(6.67 \%)$ and parental visits $(6.66 \%)$ are the main factors explaining the presence of a large flow of people in the research sector. Also, cross-border trade between Burkina Faso, Togo and Benin contributes to the development of the local economy through loading, transport of goods and taxes collected by local authorities. However, cross-border trade is marked by social, security and infrastructural constraints. It is then necessary to ask the question of whether the current actions make it possible to take advantage of the economic potential of this border area and to facilitate the crossing of populations. 
Keywords: Benin, Togo, border, issues

\section{Introduction}

Les frontières, de par leur statut de ligne de partage de deux ou plusieurs entités territoriales sont potentiellement source d'intenses activités économiques et commerciales. Elles exercent des influences sur la distribution des populations, sur l'organisation rurale et urbaine et plus concrètement sur les activités économiques (M. M. Koné, 2015, p.10). Les espaces frontaliers sont fonction des rapports d'influence entre les hommes et leur milieu. Ainsi, trois fonctions principales leur sont assignées : fonction légale, fonction fiscale et fonction de contrôle. Ces fonctions influent sur l'existence et l'organisation des groupes humains et de leurs espaces du fait de ce qu'impose cet espace (A. D. D. Nassa, 2005, p.295).

De par leur fonctionnement, les frontières contribuent à la recomposition territoriale qui s'apparentent aux pôles d'échanges, tant leur pouvoir à la fois d'attraction et de diffusion dans les contextes nationaux respectifs et au niveau régional ( S. A. Afouda, 2010, p.31). Aujourd'hui, avec la globalisation des échanges, l'intégration de la région ouest-africaine est reconnue comme un objectif stratégique majeur pour consolider le développement des économies de la sous-région et faciliter leur insertion au sein du système mondial.

Dans le secteur Matéri-Mandouri, frontière bénino-togolaise, les acteurs des échanges peuvent être classés en deux grandes catégories en fonction de leur sphère d'intervention. Certains ont pour objectif de réaliser des marges bénéficiaires à partir des transactions effectuées localement, d'autres s'occupent plus particulièrement des exportations (D. Reoungal, 2009, p.6)).

Aussi, le commerce transfrontalier relève-t-il de la fraude ou d'un commerce de contournement au sens où les acteurs s'évertuent à contourner les normes, les taxes et les règlements (K. Bennafla, 1999, p.15).

Le contexte sécuritaire de l'axe Porga-Pama en direction de Fada au Burkina-Faso a permis aux usagers de cette voie d'opérer un contournement vers la commune de Madouri. Cela intensifie le trafic des échanges entre la frontière Matéri-Mandouri et bouleverse les relations économiques transfrontalièreres.

Le fonctionnement du commerce transfrontalier dans le secteur Matéri-Mandouri fait intervenir les circuits de commercialisation et les voies d'exportation. C'est ainsi que la rivière Kpendjal sert de communication entre le Bénin et le Togo dans les échanges transfrontaliers. Ainsi, l'objectif de la présente recherche est d'étudier les enjeux de la traversée de la frontière bénino-togolaise dans le secteur Matéri-Mandouri. 


\section{Méthodologie}

\subsection{Situation géographique du milieu de recherche}

Le secteur de recherche prend en compte trois pays frontaliers (Burkina-Faso, Togo et Bénin). Les localités concernées au Burkina-Faso sont Pama, Nadiagou et Kompienga. Du côté du Togo, Koundjouaré et Mandouri sont pris en compte. Au Bénin, Dassari, Gouandé, Matéri et Tantéga sont concernés par cette recherche.

Par ailleurs, de façon spécifique, le secteur Matéri-Mandouri a fait l'objet d'analyse mais ne peut être démarqué de l'ensemble de la zone cartographiée. La zone de la présente recherche est située entre $10^{\circ} 40^{\prime}$ et $11^{\circ} 20^{\prime}$ de latitude Nord et entre $0^{\circ} 20^{\prime}$ et $1^{\circ} 20^{\prime}$ de longitude Est (Figure 1).

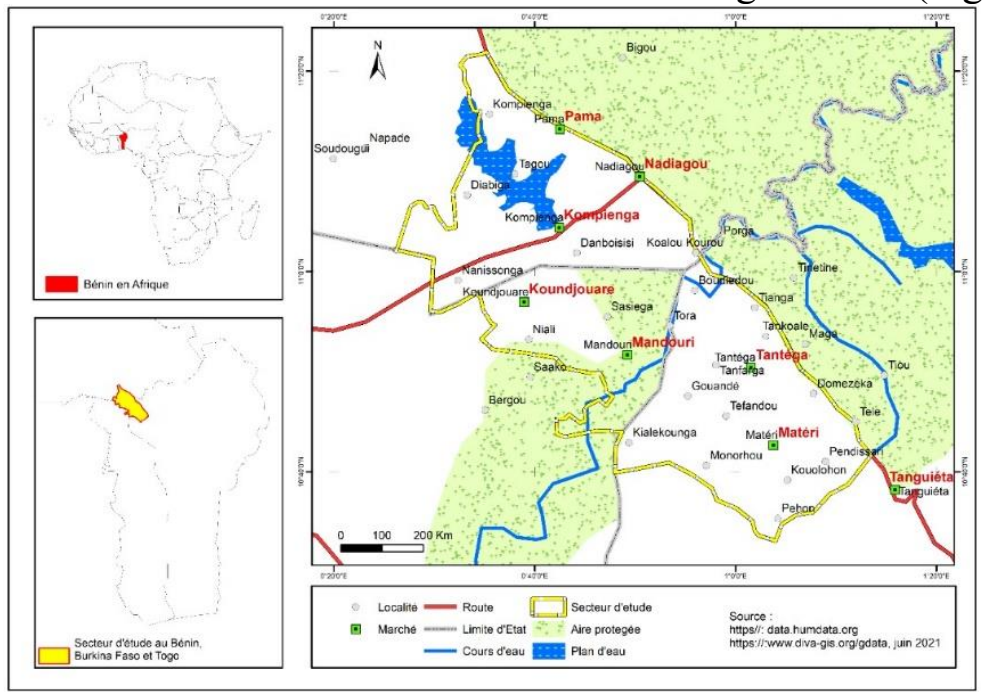

Figure 1 : Situation géographique du secteur de recherche

Le milieu de recherche se caractérise par la présence d'une frontière officielle (Porga) qui est sous la menace des actes de terrorisme grâce à sa proximité avec la partie sud-est du Burkina-Faso. C'est un espace stratégique de par sa position géographique et des échanges transfrontaliers qui s'y observent. Ils sont dominés par le commerce des produits agricoles et quelques fois des produits manufacturiers. Les produits agricoles concernent les vivriers et ceux manufacturiers concernent les vêtements, les accessoires des appareils électroniques et électriques de même que les produits artisanaux locaux.

\subsection{Collecte des données}

La collecte des données a consisté en la recherche documentaire a consisté en la collecte des données sur la mobilité transfrontalière et les travaux de terrain. Les enquêtes de terrain ont été menées auprès des populations locales (élus locaux, transporteurs, piroguiers et chargeurs), des autorités locales, des commerçants transfrontaliers et des forces de sécurité et 
de défense. Le choix de tous ces acteurs repose sur la méthode du choix raisonné qui consiste à selectionner les enquêtés capables de fournir des informations fiables et précises. Ces enquêtes ont été menées pendant la période de novembre à février. Les informations recherchées sont essentiellement liées aux gains, à la dynamique de mobilité transfrontalière et aux contraintes de la traversée de la fontière. Ainsi, le tableau 1 présente l'effectif des groupes cibles interrogés.

Tableau 1 : Répartition des personnes interrogés

\begin{tabular}{|c|c|c|c|c|}
\hline \multicolumn{2}{|c|}{ Personnes interrogées } & Bénin & Togo & Total \\
\hline \multirow{2}{*}{$\begin{array}{c}\text { Populations } \\
\text { riveraines }\end{array}$} & Piroguiers & - & 10 & 10 \\
\hline & Chargeurs & 05 & 07 & 12 \\
\hline \multirow[t]{2}{*}{$\begin{array}{l}\text { Autorités } \\
\text { locales }\end{array}$} & $\begin{array}{c}\text { Chef } \\
\text { d'Arrondissem } \\
\text { ent/Maire }\end{array}$ & 01 & 01 & 02 \\
\hline & Maire/Préfet & 01 & 01 & 02 \\
\hline \multicolumn{2}{|c|}{ Commerçants } & 30 & 35 & 65 \\
\hline \multicolumn{2}{|c|}{ Force de sécurité et de défense } & 04 & 02 & 06 \\
\hline \multicolumn{2}{|c|}{ Total } & 41 & 56 & 97 \\
\hline
\end{tabular}

Source : Enquêtes de terrain de Bakary SANOU et al., décembre 2020.

En se basant sur le choix raisonné assorti de critères comme l'activité menée par chaque acteur dans les échanges transfrontaliers et la situation socio-professionnelle, quatre-vingt-treize (93) personnes ont été prises en compte lors des travaux de terrain dont trente-huit (38) Béninois et cinquantecinq (55) Togolais.

\subsection{Traitement des données et analyse des résultats}

Le traitement des données qualitatives, des données quantitatives et des données cartographiques ont permis d'avoir des résultats fiables à travers les informations objets de recherche.

Les données quantitatives collectées ont été traitées à partir des calculs des sommes et moyennes arithmétiques des effectifs de la population et des proportions des variables utilisées. Ces calculs sont faits à l'aide des formules suivantes :

(a) $\mathrm{X}=\sum_{i=1}^{n} x i \quad$ et (b) $\mathbf{X}=\frac{\mathbf{1}}{n} \sum_{i=1}^{n} x \boldsymbol{i}$; avec n l'effectif total des acteurs enquêtés ; xi.la somme de $\mathrm{n}$ acteurs enquêtés. La formule (a) a permis de faire la somme des revenus des piroguiers, des commerçants et des motocyclistes et la formule (b) a permis de faire les moyennes liées à l'appréciation des motifs de déplacements et des contraintes de la traversée de la frontière.

Le traitement des données socio-économiques concerne l'évaluation des gains auprès des populations riveraines, des autorités locales ainsi que les commerçants. 
En ce qui concerne la matérialisation des vilages d'enquête et les marchés locaux, les coordonnées géographiques obtenues à partir du GPS ont été récupérées à l'aide du logiciel MapSource, puis transférées dans le logiciel QGIS 9.3 qui a servi à la réalisation de la carte montrant la spatialisation des circuits frauduleux dans le secteur de recherche. Cette approche méthodologique a permis d'aboutir aux résultats qui se présentent comme ciaprès.

\section{Résultats}

\subsection{Motifs de la traversée de la frontière}

La plupart des frontières sont des endroits qui offrent des opportunités d'échange. Elles constituent donc des lieux stratégiques où il est enregistré un flux important de personnes. Ainsi, plusieurs raisons justifient la fréquentation massive de ces frontières (figure 2).

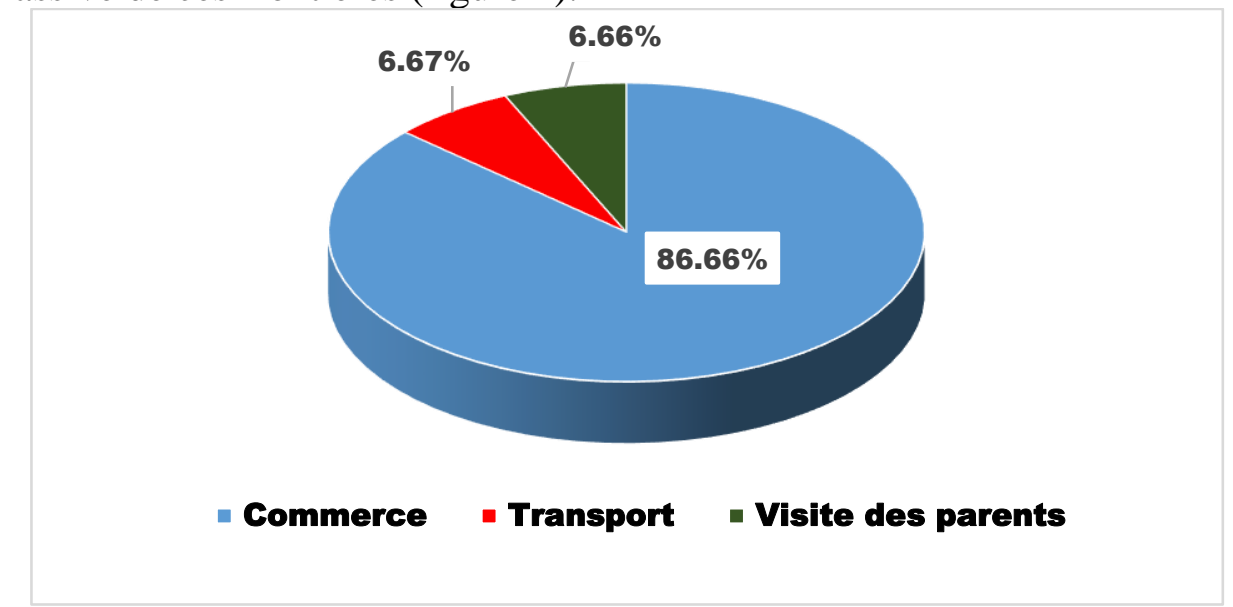

Figure 2 : Motifs de la traversée de la frontière bénino-togolaise dans le secteur MatériMandouri

Source : Travaux de terrain, décembre 2020.

Il ressort des résultats que $86,66 \%$ de personnes traversent la frontière pour raison de commerce. Les échanges transfrontaliers s'organisent pour la plupart autour d'un réseau de marchés bien structuré servant ainsi de canaux d'importation et d'exportation des produits de diverses natures. Les marchés qui attirent cet important flux du côté du Bénin sont: Gouandé, Matéri et Tantéga. Dans la partie togolaise, il s'agit des marchés de Mandouri et de Koundjouaré. Outre cet aspect commercial, 6,67 \% des personnes traversent cette frontière pour motif de transport de marchandises. Enfin, les populations du secteur Gouandé-Mandouri étant liés par un métissage culturel, 6,66 \% affirment qu'ils traversent la frontière pour la visite des parents. 
Dans le commerce transfrontalier, plusieurs produits font objet d'échange (planche 1) notamment à la frontière bénino-togolaise dans le secteur Gouandé-Mandouri.

Photo 1 a photo $1 b$

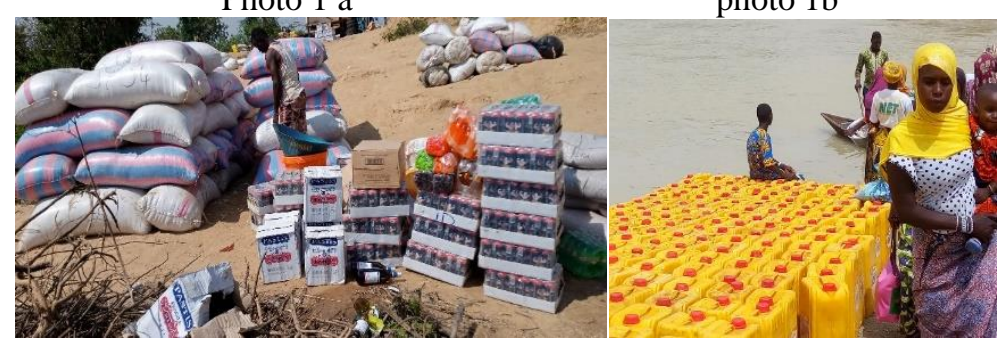

Planche 1 : Quelques produits d'échanges dans le secteur de recherche

Prise de vue : B. Sanou, mars 2020.

En observation la planche 1, il ressort que plusieurs produits traversent la frontière du secteur de recherche. Il s'agit majoritairement des produits agricoles $(54,28 \%)$ et des produits manufacturiers $(45,72 \%)$. Les produits agricoles traversés sont les céréales, les tubercules et racines et les légumineux. S'agissant des produits manufacturiers, ils concernent les vêtements, les panneaux solaires et les produits agroalimentaires. La traversée de ces produits représente des avantages non seulement pour les populations riveraines mais aussi pour les commerçants et autorités locales.

\subsection{Activités menées à la traversée de la frontière}

Les échanges transfrontaliers créent d'énormes avantages à divers acteurs. Ainsi, le secteur Gouandé-Mandouri abrite des marchés qui intensifient des échanges. La planche 2 montre les différents moyens et les activités créés lors de la traversée de la rivière Kpendjal ainsi que les différents bénéficiaires.

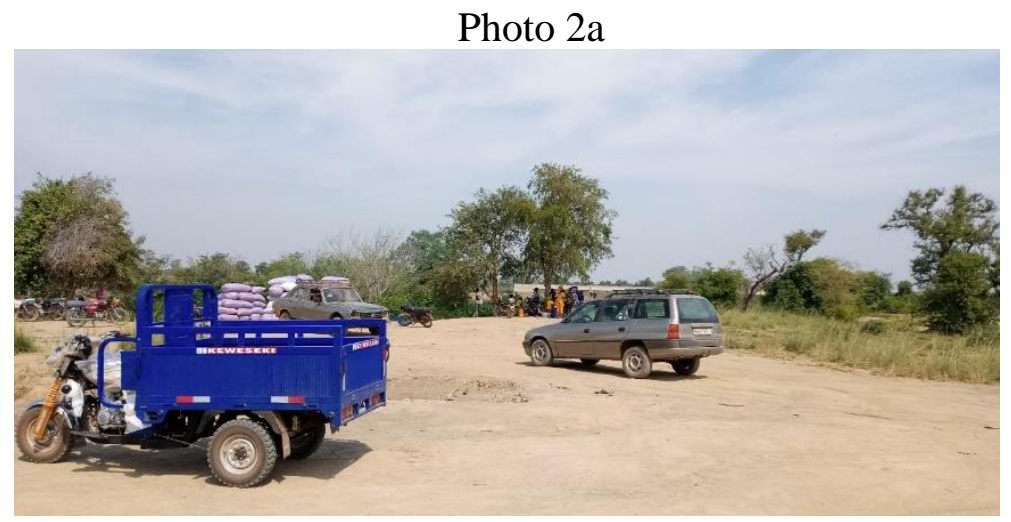




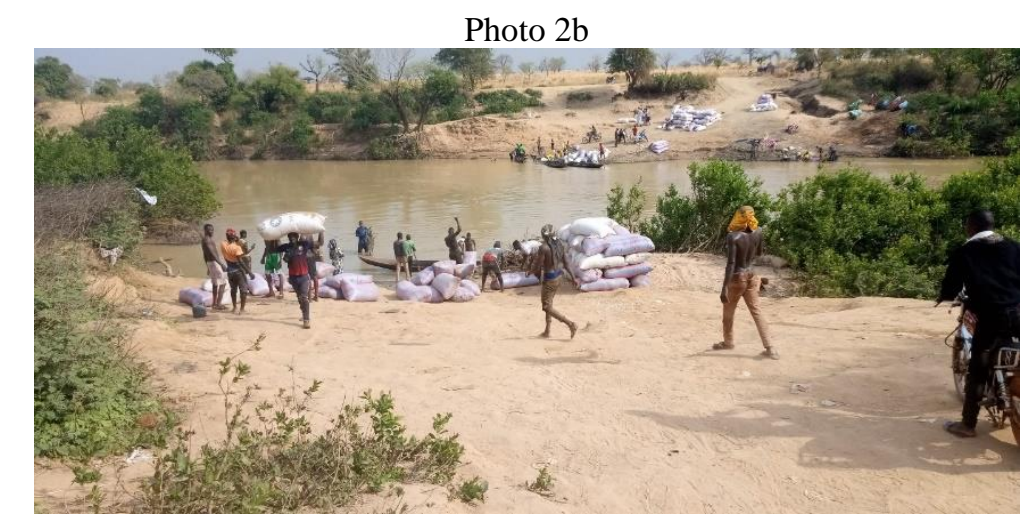

Planche 2 : Avantages de la traversée de la frontière sur la rivière Kpendal dans le secteur Mandouri (Togo)-Gouandé (Bénin)

Prise de vue : B. Sanou, janvier 2021.

La planche 2 montre de multiples avantages offerts aux populations lors de la traversée de la rivière des produits marchands. Ces avantages concernent la création d'emploi pour les jeunes de la localité. Les principaux bénéficiaires sont les populations locales, les autorités locales et les commerçants.

En effet, les bénéficiaires dans le rang des populations locales concernent les transporteurs, les chargeurs et les piroguiers.

Pour apprécier la rentabilité des opportunités créées par les échanges transfrontaliers, les revenus moyens saisonniers de certains acteurs clés du système ont été présentés. Ces revenus ont concerné les gains pendant la petite saison et la grande saison. Les saisons représentent ici, les périodes de petites recettes ou de grandes recettes. Ces périodes ne sont pas définies en termes de mois de l'année mais les moments de fortes mobilisations de recettes et de faibles mobilisations de recettes. La figure 3 présente les revenus moyens obtenus par acteur lors des travaux de terrain. 


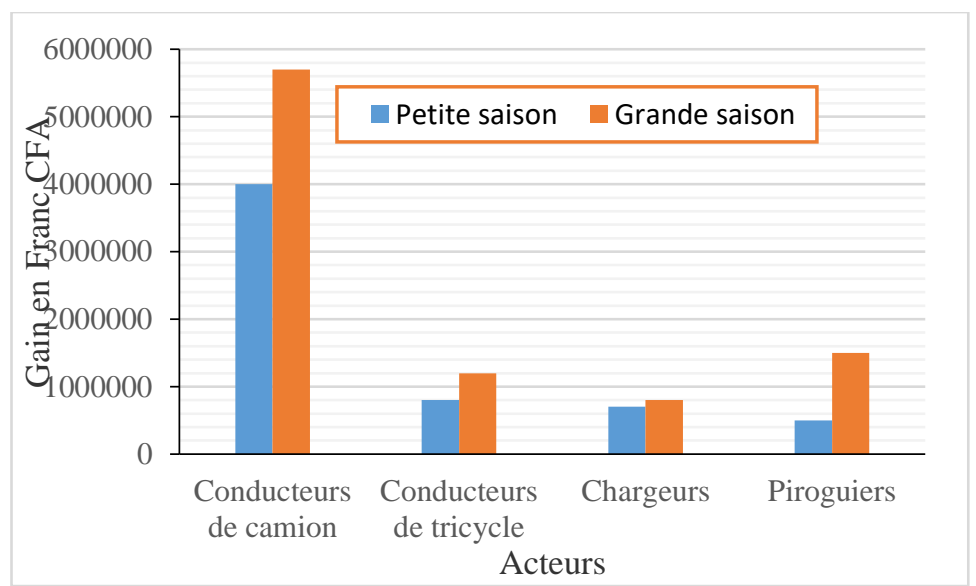

Figure 3 : Revenus moyens par saison des échanges de quelques acteurs

Source : Travaux de terrain, décembre 2020.

Après l'examen de la figure 3, il est à retenir que pendant la grande saison, les conducteurs de camion atteignent en moyenne 5600000 FCFA et en petite saison 4000000 FCFA soit un manque à gagner de 1600000 FCFA. En revanche, au cours de la grande saison, les conducteurs de tricycle, les piroguiers et les chargeurs n'arrivent pas à dépasser 1500000 FCFA.

En effet, les transporteurs (conducteurs de camions et de tricycle) sont ceux qui s'occupent du transport des marchandises. Quant aux chargeurs, ils ont pour rôle le chargement et le déchargement des marchandises lors de la traversée de la rivière. Enfin, les piroguiers eux, ils sont chargés de faire traverser les personnes et leurs biens à l'aide de pirogues.

Par ailleurs, pour contourner l'axe officiel, où le pont de Kpendjal est actuellement en construction, les axes frauduleux sont créés pour développer la contrebande. Ces axes permettent aux contrebandiers d'échapper aux contrôles douaniers qui les obligent à payer les taxes. Ils permettent également de contourner la frontière officielle de Porga où les taxes sont perçues par les agents de sécurité publique. Le point d'entrée officiel entre la commune de Matéri et celle de Mandouri est le pont reliant les deux localités, actuellement en construction. A travers ces axes, les commerçants arrivent à faire traverser les produits manufacturés en provenance du Burkina et du Togo notamment le marché international de Cinkansé.

Quand bien même que les échanges transfrontaliers offrent des opportunités à certains acteurs dans le secteur de recherche, il existe également des contraintes. 


\subsection{Contraintes liées à la traversée de la frontière dans le secteur de recherche}

Les contraintes liées à la traversée de la frontière bénino-togolaise dans le secteur Gouandé-Mandouri sont multiples. Ils sont d'ordre social, sécuritaire et infrastructurel.

S'agissant des contraintes d'ordre social, on note les conflits entre étrangers et autochtones de la localité traversée ; le rançonnement de la part des acteurs publics de contrôle et de sécurité ; la surtaxe sur les marchandises.

Sur l'appréciation des contraintes d'ordre sécuritaire, les avis sont partagés auprès des personnes interrogées (figure 4).

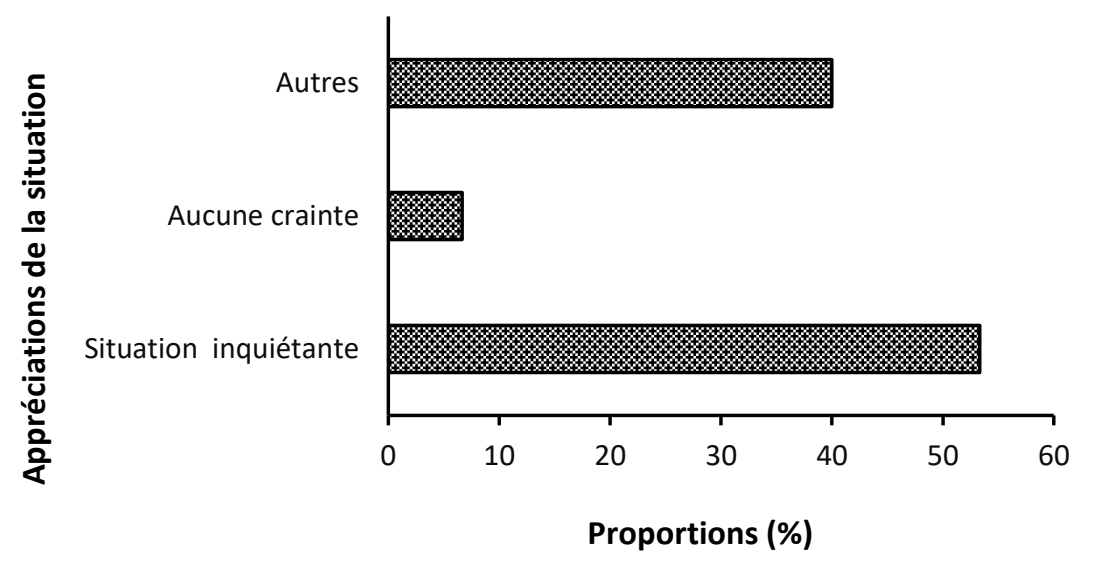

Figure 4: Appréciations de la situation sécuritaire dans le secteur Gouandé-Mandouri Source : Enquêtes de terrain, décembre 2020.

De l'analyse de la figure 4 , il ressort que $53,33 \%$ des enquêtés déclarent que la situation sécuritaire est inquiétante dans le secteur de recherche contre $6,67 \%$ estimant qu'il n'y a aucune crainte. Toutefois, $40 \%$ de ces derniers sont restés sans réponse. Autrement dit, la catégorie de "autres" " est ceux qui se sont de façon volontaire réservés de s'exprimer pour des raisons de leur propre sécurité. De façon globale, l'insécurité constitue une contrainte majeure dans les échanges transfrontaliers du secteur de recherche.

Outre ces contraintes, d'autres contraintes pèsent sur les échanges dans le secteur de recherche. Il s'agit des contraintes d'ordre infrastructurel. La photo 5 montre le calvaire de la traversée de la frontière bénino-togolaise dans le secteur Gouandé-Mandouri. 


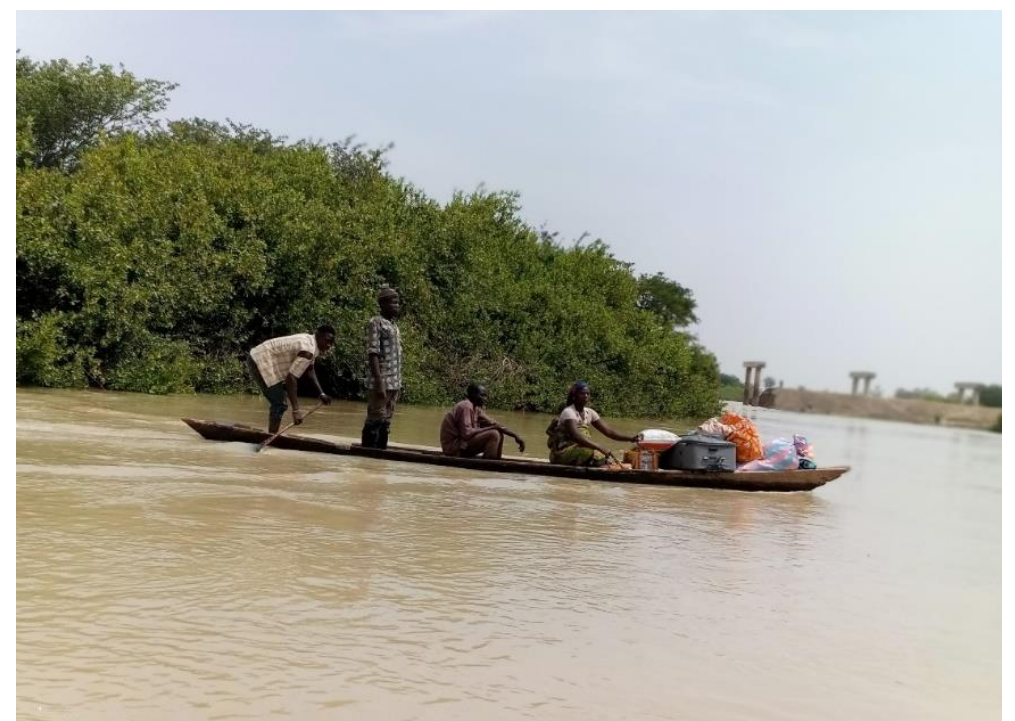

Photo 5 : Traversée de la frontière bénino-togolaise dans le secteur Gouandé-Mandouri Prise de vue : B. Sanou, décembre 2020.

En quête de voies et moyens pour la libre circulation des personnes et des biens entre le Bénin, le Burkina-Faso et le Togo, les populations transfrontalières s'évertuent à traverser la frontière bénino-togolaise à travers les pirogues par la rivière Kpendjal. Cette situation est due par le fait que le pont qui reliait les deux pays a été endommagé il y a plus de cinq (05) ans. Ainsi, sa construction a débuté depuis 2017 et jusque là, l'ouvrage est en chantier. Ce qui constitue un handicap aux échanges transfrontaliers. De même, cette traversée est très risquée du fait de l'état des pirogues et les surcharges que font les piroguiers qui ne mesurent parfois pas les risques encourus. La traversée est conditionnée par le débit de l'eau qui est toujours fort même pendant l'étiage.

\subsection{Mesures palliatives aux contraintes}

Face aux contraintes dont vivent les commerçants transfrontaliers, des mesures palliatives ont été proposées. Par rapport aux conflits transfrontaliers, il a été envisagé de mettre en place un comité mixte de gestion de conflits dans le secteur de recherche; aux fins de remédier aux tracasseries routières et de lutter efficacement contre le rançonnement.

En ce qui concerne l'insécurité, il urge d'organiser des patrouilles conjointes entre forces de sécurité et de défense du Bénin, du Togo et du Burkina-Faso.

S'agissant des contraintes d'ordre infrastructurel, Il convient dans la partie béninoise, d'aménager l'axe Matéri-Gouandé-Frontière. En ce qui concerne la partie togolaise, des démarches sont en cours au niveau préfectoral 
pour négocier avec le gouvernement en vue de prendre ses responsabilités pour la finalisation du pont en construction par l'entreprise EBOMAF sur la rivière Kpendjal et faciliter le trafic.

\section{Discussion}

Les échanges transfrontalières dans le milieu de recherche sont principalement liés au commerce, au transport de marchandises et à la visite des parents vu le lien culturel entre les populations transfrontalières du secteur de recherche.

En effet, les produits échangés à travers cette frontière sont les produits vivriers et les produits manufacturés. Les présents résultats corroborent ceux obtenus par A. D. D. Nassa (2005, p.99) qui ont trouvé que les échanges frontaliers partent du commerce capillaire, basé sur les produits locaux agricoles, à un commerce de longue distance qui se caractérise par l'importance de son volume et par le nombre de personnes qui y participent. A travers ce commerce, les populations riveraines des zones d'échange bénéficient de plusieurs avantages. Ces avantages concernent entre autre la traversée de la rivière Kpendjal par la pirogue et aux chargements des marchandises contribuant ainsi au développement économique local de ces populations. Ainsi, les travaux de R. Nkendah et al. (2012, p.40) ont montré que le transfert du plantain à partir des marchés frontaliers du sud Cameroun vers le Gabon et la Guinée Équatoriale génère des recettes à divers acteurs. Elles découlent du transport, de la manutention et du déplacement de la marchandise.

Les contraintes liées aux échanges transfrontaliers dans le milieu de recherche sont entre autres : les rançonnements de la part des acteurs publics de contrôle et de sécurité et la surtaxe sur les marchandises de la part des autorités locales, les contraintes d'ordre sécuritaire liées aux attaques terroristes et aux braquages, les contraintes d'ordre infrastructurel liées à l'impraticabilité des routes voire même leur inexistence. Les résultats similaires ont été obtenus par PROFAB (2017, p.21) qui estime que les femmes impliquées dans le commerce transfrontalier sont confrontées à plusieurs problèmes dont : la faible disponibilité des infrastructures du commerce (routes, magasins, hangars), l'inadéquation des transports publics et privés, les prélèvements douaniers et autres paiements, la médiocrité de la sécurité, le manque de compétences en gestion des entreprises et le faible accès à l'information, l'analphabétisme et le faible niveau d'instruction des femmes le harcèlement des femmes de la part des agents de contrôles et la corruption. Dans les échanges transfrontaliers et développement local au Nord-ouest de l'Atacora, I. Tadja $(2020$, p.129) a trouvé aussi que plusieurs difficultés entravent les échanges transfrontaliers. Les plus couramment évoquées, sont 
celles relatives aux tracasseries administratives, aux services de contrôle et de sécurité publique.

\section{Conclusion}

La présente recherche portant sur les enjeux de la traversée transfrontalière a permis de retenir que plusieurs raisons expliquent la présence d'un important flux de personnes dans le secteur Gouandé-Mandouri. La présence de ces dernières est liée majoritairement au commerce. Les échanges transfrontaliers entre le Burkina-Faso, le Togo et le Bénin offrent nombreux avantages aux populations locales. Les autorités locales en bénéficient à travers les taxes perçues auprès des commerçants.

Cependant, les échanges transfrontaliers sont marqués par des contraintes d'ordre social, sécuritaire et infrastructurel. A cet effet, il urge alors que les mesures palliatives soient trouvées. Il s'agit de mettre en place un comité mixte de gestion de conflits dans le secteur de recherche; de remédier aux tracasseries routières, de lutter efficacement contre le rançonnement et d'organiser des patrouilles conjointes entre forces de sécurité et de défense du Bénin, du Togo et du Burkina-Faso. La coopération transfrontalière doit ainsi être revue afin de faciliter les échanges entre ces frontières.

\section{References:}

1. AFOUDA Alix Servais, 2010, Echanges transfrontaliers et organisation de l'espace dans le bassin du lac Tchad. Thèse de doctorat en Géographie, Université Michel de Montaigne, Bordeaux III, 405 p.

2. BENNAFLA Karine,1999, La fin des territoires nationaux ? État et commerce frontalier en Afrique centrale. Politique africaine $n^{\circ} 73,26$ p.

3. MAMADOU KONE Mahanam Moustapha, 2015, Marchands ouestafricains et marchés frontaliers : construction d'une communauté d'affaires. Thèse de doctorat en Géographie. Université Michel de Montaigne - Bordeaux III, $265 \mathrm{p}$.

4. NASSA Dabié Désiré Axel, 2005, Commerce transfrontalier et structuration de l'espace au nord de la Côte d'Ivoire. Thèse de doctorat : Géographie : Université Michel de Montaigne-Bordeaux 3, 336 p.

5. NASSA Dabié Désiré Axel, 2008, Dynamisme d'une ville stimulé par la frontière : l'exemple d'Ouangolodougou au Nord de la Côte d' Ivoire. Territoire en mouvement. Revue de géographie et aménagement, $12 \mathrm{p}$.

6. NKENDAH Robert, AKO Edouard, TAMOKWE Bertrand, NZOUESSIN Chantal, NJOUPOUOGNIGNI Moussa, MELINGUI 
Estelle et Azeufouet Alain, 2012, Le commerce transfrontalier informel des produits agricoles et horticoles, Économie rurale, 34-49.

7. PROFAB, 2017, Programme d'appui pour la libre circulation des produits agricoles en Afrique de l'ouest. Rapport d'étude.

8. REOUNGAL Djinidjil, 2009, Aperçu sur les grandes tendances des échanges transfrontaliers de produits agricoles entres le Tchad et les pays limitrophes. ITRAD, N'Djaména, $10 \mathrm{p}$.

9. TADJA Idrissou, 2020, Echanges transfrontaliers et développement local au Nord-ouest de l'Atacora au Bénin. Thèse de doctorat en Géographie, Université d'Abomey-Calavi, 183 p. 